\title{
USO DE LA EVIDENCIA EN POLIITICAS Y PROGRAMAS DE SALUD APORTES DEL INSTITUTO NACIONAL DE SALUD
}

\author{
Ericson L. Gutierrez ${ }^{1, a}$, Marina Piazza ${ }^{1, b}$, Alfonso Gutierrez-Aguado,a, Gisely Hijar ${ }^{1, \mathrm{c}}$, Gloria Carmona ${ }^{1, \mathrm{~d}}$, \\ Patricia Caballero, ${ }^{1, \mathrm{e}}$, Nora Reyes ${ }^{1, \mathrm{f}}$, Carlos Canelo ${ }^{1, \mathrm{~g}}$, Juan Pablo Aparco ${ }^{1, \mathrm{~h}}$, Romina A. Tejada ${ }^{1, \mathrm{f}}$, \\ Rafael Bolaños-Díaz ${ }^{1, g}$, Silvia Saravia ${ }^{1, a}$, Ernesto Gozzer ${ }^{1, f}$
}

\begin{abstract}
RESUMEN
En el presente artículo se analizan algunos ejemplos del uso de evidencia generada por el Instituto Nacional de Salud (INS) que el Ministerio de Salud del Perú ha realizado en los últimos años en la formulación, implementación y evaluación de las políticas y programas. Presenta el proceso por el cual los programas presupuestales se sustentan y fortalecen a partir de la evidencia. Describe el progreso en el desarrollo de una metodología para generar guías de calidad a partir de la mejor evidencia disponible para orientar la práctica clínica. Presenta algunos ejemplos de requerimientos de evidencia del Ministerio de Salud al Instituto Nacional de Salud y su impacto en política. Por último, propone direcciones futuras respecto a metodologías de investigación especialmente relevantes para el desarrollo y evaluación de políticas y el fortalecimiento de redes de evaluación de tecnologías en el ámbito nacional e internacional.
\end{abstract}

Palabras claves: Práctica clínica basada en evidencia; Evaluación; Tecnología sanitaria; Política pública; Toma de decisiones; Perú (Fuente: DECS BIREME).

\section{USE OF EVIDENCE IN HEATH POLICIES AND PROGRAMS CONTRIBUTIONS OF THE INSTITUTO NACIONAL DE SALUD}

\begin{abstract}
This article analyzes some examples about how the Ministry of Health of Peru has used evidence for policy and program formulation, implementation and evaluation. It describes the process by which health budget programs are based and strengthened with scientific evidence. Provides an overview about how the development of clinical guidelines methodology is facilitating the generation of high quality evidence based clinical guidelines. It presents some examples of specific information needs of the Ministry of Health to which the Instituto Nacional de Salud has responded, and the impact of that collaboration. Finally, the article proposes future directions for the use of research methodology especially relevant for the development and evaluation of policy and programs, as well as the development of networks of health technology assessment at the national and international level.
\end{abstract}

Key words: Evidence-based medicine; Evaluation; Technology assessment; Public policy; Decision-making; Peru (Source: MeSH NLM).

\section{INTRODUCCIÓN}

La política basada en evidencias busca fundamentar su desarrollo a partir de conocimiento generado con un alto grado de rigurosidad. La evidencia puede sustentar el proceso desde la identificación del problema, la formulación, y la implementación de la política. Asimismo, puede determinar el impacto de la política y los ajustes necesarios para mejorar sus resultados y aplicabilidad en distintas poblaciones. Este enfoque, coloca la mejor evidencia científica disponible en el centro del desarrollo e implementación de las políticas ${ }^{(1,2)}$.

El Instituto Nacional de Salud (INS), como Organismo Público Ejecutor del Ministerio de Salud (MINSA), tiene como misión la promoción, desarrollo y difusión de la investigación científica. En ese sentido, el INS fomenta el desarrollo de investigaciones con impacto en salud pública, promoviendo la creación de evidencia científica necesaria para prevenir y controlar las principales

\footnotetext{
Instituto Nacional de Salud. Lima, Perú.

Ministerio de Economía y Finanzas. Lima, Perú.

Médico especialista en gestión en salud; ${ }^{\mathrm{b}}$ psicóloga clínica, MPH, Sc.D.; ${ }^{\mathrm{c}}$ bióloga, MSc; ${ }^{\mathrm{a}}$ obstetriz, MSc, ${ }^{\mathrm{e}}$ médico infectólogo, MSc; ${ }^{\mathrm{f}}$ médico cirujano, MSc;

${ }^{\mathrm{g}}$ médico internista, MSc; ${ }^{\mathrm{h}}$ nutricionista

Recibido: 14/05/2016 Aprobado: 06/07/2016
}

Citar como: Gutierrez EL, Piazza M, Gutierrez-Aguado A, Carmona G, Caballero P, Reyes N, et al. Uso de la evidencia en políticas y programas de salud aportes del Instituto Nacional de Salud. Rev Peru Med Exp Salud Publica. 2016;33(3)580-4. doi:10.17843/rpmesp.2016.333.2308 
enfermedades que afectan a la población. La Unidad de Análisis y Generación de Evidencias en Salud Pública (UNAGESP) del INS, se encarga de proponer recomendaciones para el desarrollo de política de salud a partir de la generación y análisis de evidencia científica. Es una unidad pionera en implementar la cultura del conocimiento y el uso sistemático de las evidencia en el sistema público nacional de salud para mejorar la calidad de las políticas públicas.

\section{EVIDENCIA PARA SUSTENTAR PROGRAMAS PRESUPUESTALES}

EI MINSA ha aplicado evidencia en la fundamentación de sus programas presupuestales. El presupuesto por resultados vincula la asignación de recursos a bienes y servicios, definiendo resultados medibles para mejorar las condiciones de vida de la población. Un programa presupuestal, principal instrumento de presupuesto por resultado, es diseñado para resolver un problema específico, puede involucrar diferentes sectores y niveles de gobierno y sus actividades se ejecutan a nivel nacional, regional y local ${ }^{(3)}$. El diseño del programa presupuestal se inicia con la identificación de un problema y una población. A partir de un análisis causal de los factores que generan el problema se define el resultado que busca generar el programa. Seguidamente, se busca evidencia acera de los medios para revertir el problema. Estos medios o alternativas de solución se evalúan comparando la calidad de la evidencia, la efectividad y la viabilidad de las alternativas. Por último, las alternativas se traducen en productos que recibe la población objetivo, con el fin de lograr un cambio ${ }^{(4)}$. En otras palabras, los bienes y servicios que se proporciona a la población son seleccionados a partir de demostrada efectividad para resolver los problemas de salud.

Cada fase del diseño de un programa presupuestal debe basarse en la mejor evidencia disponible con una clara estrategia de búsqueda de información. Los estudios son seleccionados de acuerdo a una jerarquía de metodologías con distinta capacidad para identificar causalidad entre las variables de interés. Por ejemplo, tienen mayor nivel de evidencia el metaanálisis, revisiones sistemáticas y estudios experimentales aleatorizados, que los estudios observacionales. Por ejemplo, con esta metodología se generó el Programa Articulado Nutricional con el objetivo de reducir la desnutrición crónica en niños menores de 5 años. Así, recientemente, una evaluación de impacto mostró que los niños nacidos después de la aplicación de este programa tuvieron $5 \%$ menos de probabilidad de presentar desnutrición crónica ${ }^{(5)}$.

Los programas presupuestales una vez implementados requieren ser monitoreados con indicadores de desempeño en estudios poblacionales. Con el objetivo de monitorear y evaluar los programas presupuestales el MINSA desarrolló, con el Instituto Nacional de Estadística e Informática, seis encuestas poblacionales: Encuesta Nacional de Hogares (6), Encuesta de Demografía y Salud Familiar ${ }^{(7)}$, Encuesta de Programas Presupuestales ${ }^{(8)}$, Encuesta de Establecimientos de Salud ${ }^{(9)}$, Encuesta de Crecimiento y Desarrollo ${ }^{(10)}$ y la Encuesta de Riesgo Anual de Infección a Tuberculosis (11). EI INS participó en la identificación de indicadores e instrumentos de recolección de información para este fin.

\section{METODOLOGÍA DE GUÍAS PARA ORIENTAR LA PRÁCTICA CLIINICA}

Por otro lado, el MINSA ha elaborado una metodología que permite basar la práctica clínica en la evidencia. Para ello, elaboró con el INS la metodología para desarrollar las Guías de Práctica Clínica (GPC) del sector Salud en el $2015^{(12)}$. Las GPC son un conjunto de recomendaciones basadas en revisiones sistemáticas para tomar decisiones sobre las alternativas diagnósticas o terapeuticas frente a una condición clínica o problema de salud ${ }^{(12)}$. La Guía Metodológica para la Elaboración de GPC tiene como objetivo estandarizar la metodología para la generación de guías de calidad en establecimientos de salud basadas en la mejor evidencia. La Norma Técnica de Salud para la Elaboración y Uso de Guías de Práctica Clínica del Ministerio de Salud, igualmente fue aprobada por el MINSA en el $2015^{(13)}$ y ha permitido la generación de nuevas guías como la Guía de Práctica Clínica para el Diagnóstico, Tratamiento y Control de la Diabetes Mellitus Tipo 2 en el primer nivel de atención (13) y la de dengue, en fase final de aprobación por el MINSA.

\section{EVIDENCIA PARA TOMAR DECISIONES DE POLÍTICAS Y PROGRAMAS NACIONALES}

EI MINSA también ha hecho uso de evidencia científica para ajustar programas existentes, por ejemplo, la Estrategia Nacional de Alimentación y Nutrición Saludable del MINSA a través del Plan de Lucha contra la Desnutrición y Reducción de la Anemia, requirió al INS determinar la efectividad de una intervención comunitaria para mejorar la adherencia a la suplementación con micronutrientes en polvo. El estudio identificó barreras a la suplementación en distintos niveles. En establecimientos de salud se encontró desabastecimiento de micronutrientes y una limitada capacidad de distribución del suplemento utilizando exclusivamente el Servicio de Crecimiento y Desarrollo (CRED). En la comunidad y sus líderes, el estudio observó falta de información y una percepción negativa del micronutriente. En el contexto del hogar se 
percibió desconfianza hacia el uso del micronutriente, rechazo al sabor del producto y se puso en evidencia falta de conocimiento acerca de cómo usarlo. Las recomendaciones generadas por el estudio permitieron modificar en el 2016 la Directiva Sanitaria de Suplementación 056 por la 068 que incluye la ampliación de ventanas de entrega del micronutriente en los establecimientos de salud e incorpora agentes comunitarios en el seguimiento del consumo de micronutrientes ${ }^{(15)}$.

Otro ejemplo de uso de evidencia en política de salud se refiere a la necesidad del MINSA de determinar los efectos adversos de los alimentos genéticamente modificados (AGM) en la salud humana. La revisión sistemática desarrollada por el INS concluyó que la evidencia científica disponible no resultaba suficiente para determinar que el consumo de los AGM no genera efectos adversos en la salud humana. Esta revisión determinó, asimismo, la necesidad de desarrollar estudios de evaluación de la inocuidad de los AGM en humanos y animales de acuerdo a los estándares científicos internacionales ${ }^{(16)}$. En consecuencia, se promulgó la Ley 29811 y su reglamento que establece la moratoria al ingreso y producción de organismos vivos modificados (OVM o transgénicos) al territorio nacional por un periodo de 10 años. La finalidad de la moratoria es la de fortalecer capacidad nacional en bioseguridad, regular el ingreso de OVM a nuestro país y minimizar el riesgo generado por la aplicación de la biotecnología relacionada con los OVM, cuidando el ambiente, las especies nativas, la agrobiodiversidad y recursos genéticos nativos ${ }^{(17)}$.

EI MINSA ha sustentado en conocimiento importantes decisiones en inmunizaciones. Por ejemplo, el Perú contaba con un esquema de vacuna contra siete serotipos para prevenir la enfermedad neumocócica, pero su producción se iba a discontinuar. Por tanto, se requería elegir entre una vacuna contra diez serotipos (VCP10) y otra contra trece serotipos (VCP13). En respuesta a esta necesidad de evidencia del MINSA el INS realizó dos evaluaciones económicas. El primer estudio demostró que la vacuna VCP13 es más costo efectiva que la VCP10 para prevenir neumonía en niños menores de cinco años en Perú ${ }^{(18,19)}$. El segundo estudio extendió el análisis a otras afecciones: otitis media y meningitis encontrando similares resultados ${ }^{(20,21)}$. A raíz de estos resultados se decidió incluir la vacuna VCP13 en el Programa Nacional de Inmunizaciones en reemplazo de la vacuna VCP7 ${ }^{(22)}$.

De igual manera, la Estrategia Sanitaria Nacional de Inmunizaciones en el esquema de vacunación contra el papiloma virus incluía la vacuna bivalente (contra serotipos 16 y 17), y requería contar con evidencia acerca de su eficacia. Por tanto, necesitaba conocer si se debía seguir incluyendo esta vacuna en el esquema de vacunación y comparar su eficacia con la vacuna cuadrivalente (contra serotipos $6,11,16$ y 18 ). En respuesta, se implementaron tres revisiones sistemáticas sobre la eficacia de la vacuna comparando la bivalente con la cuadrivalente. La primera revisión comparó ambas vacunas respecto a su eficacia en la prevención de lesiones premalignas y cáncer de cuello uterino ${ }^{(23)}$. La segunda revisión comparó ambas vacunas respecto a su eficacia para prevenir lesiones genitales premalignas y cáncer no uterino ${ }^{(24)}$. La tercera revisión se propuso evaluar la eficacia de estas vacunas en prevenir verrugas ano-genitales. Se evidenció que ambas vacunas tenían similar eficacia en la prevención de lesiones premalignas y cáncer de cuello uterino. Solo se encontraron reportes de eficacia de la vacuna cuadrivalente en la prevención de cáncer genital no uterino y de verrugas ano-genitales. Los resultados evidenciaron eficacia para la prevención de verrugas, así como de neoplasia intraepitelial anal en hombres que tienen sexo con hombres ${ }^{(25)}$. Luego, desde el punto de vista económico, se desarrolló una evaluación costo-efectividad que demostró que ambas vacunas eran equivalentes ${ }^{(26)}$. En consecuencia, el MINSA tomó la decisión de cambiar de la vacuna bivalente a la cuadrivalente.

El MINSA requería evaluar la posibilidad de incluir el tratamiento antiviral gratuito para los casos de hepatitis B crónica diagnosticados y sin acceso a terapia específica. Desde la perspectiva del mejor uso de recursos económicos, carecía de información para elegir entre distintos tratamientos. EI INS revisó la evidencia disponible acerca de costo-efectividad de regímenes antivirales para el tratamiento de hepatitis $B$ crónica. El estudio determinó que el tratamiento antiviral para hepatitis B crónica es una intervención costoefectiva en muchos sistemas de salud. Se identificó que existe variabilidad en el costo-efectividad de los distintos esquemas y se recomendó generar información local para compararlos. En consecuencia, de este estudio el MINSA tomó la decisión de incluir tratamiento antiviral para los casos de hepatitis B crónica ${ }^{(27)}$.

En el marco de los lineamientos de los derechos de la salud sexual y reproductiva, el MINSA pone a disposición de la población diversos métodos anticonceptivos (28). En concordancia al objetivo de reducción de mortalidad materna del Plan Nacional Concertado de Salud, a partir del año 2001 el MINSA incorporó el uso de la anticoncepción oral de emergencia en la Norma de Planificación Familiar (29). Esta incluye métodos hormonales que se usan luego de una relación sexual sin protección, para evitar un embarazo no deseado. Dada la controversia acerca del mecanismo de acción del levonorgestrel como anticonceptivo oral de emergencia; el INS respondió al requerimiento de información del MINSA 
desarrollando una revisión sistemática. El estudio concluyó que el principal mecanismo de acción del levonorgestrel en las dosis recomendadas como AOE, es la inhibición o retraso de la ovulación, sin afectar la capacidad de los espermatozoides de migración y penetración al óvulo. El estudio determinó que no existe evidencia de alteraciones morfológicas ni moleculares en el endometrio que puedan interferir con la implantación del huevo fecundado. Es decir, el estudio concluyó que no existe evidencia científica acerca de un posible efecto abortivo del levonorgestrel como anticonceptivo oral de emergencia ${ }^{(30)}$.

\section{EVIDENCIA EN PROGRAMAS REGIONALES}

Un ejemplo interesante del uso de la evidencia en un programa de carácter regional es el de la vacunación por rabia silvestre en Amazonas. Desde 1975 al 2010 se notificaron más de 250 muertes por esta enfermedad, la mayoría ocurrieron en menores de 15 años de edad de comunidades nativas de la etnia awajún en la provincia de Condorcanqui. En en el año 2011, el MINSA inició una campaña de vacunación en dicha provincia, que tuvo una limitada implementación por falta de aceptabilidad de la vacuna en esta comunidad. En respuesta al requerimiento del MINSA, el INS realizó un estudio que tuvo como objetivo conocer la percepción sobre la vacunación contra la rabia silvestre en comunidades nativas de la etnia awajún ${ }^{(31)}$. Utilizó una metodología cualitativa en la población, autoridades locales y personal de salud. Las percepciones recogidas hacia este tipo de vacunación manifiestan una falta de claridad respecto a su potencial beneficio o necesidad para la comunidad, relacionada a cuestiones religiosoculturales, a las limitadas condiciones socio-económicas así como a su fragmentada comunicación con el estado y el sistema de salud peruano. El informe fue incorporado en la estrategia comunicacional sobre rabia silvestre del MINSA del $2013^{\left({ }^{(3)}\right)}$ y presentado y discutido con la Dirección de Salud de Amazonas y a las con dirigentes locales y regionales de Amazonas, Condorcanqui y Chachapoyas.

\section{CONCLUSIONES}

El presente artículo ha presentado algunos ejemplos de utilización de evidencia en el diseño y mejora de políticas y programas de salud. Es importante notar que el contexto de la agenda para el desarrollo sostenible y de la extensión de la cobertura universal en salud, plantean nuevas necesidades de generación y uso de la evidencia. Por tanto, se requiere responder a preguntas críticas en el ejercicio de la política y la implementación de programas de salud pública. Algunas metodologías especialmente relevantes para ello son, estudios de impacto de intervenciones, estudios de impacto presupuestario, estudios de eficiencia técnica, eficiencia económica y estudios de factibilidad de implementación de intervenciones exitosas, entre otras. Un mecanismo para el desarrollo de este trabajo es la oportunidad de proponer investigación enmarcada en los programas presupuestales.

Es necesario reconocer que la cultura de toma de decisiones basadas en información es reciente en nuestro país y que la demanda de información se ha acelerado en forma importante. Por tanto, resulta fundamental la coordinación entre las instituciones nacionales e internacionales que desarrollan evaluación de tecnologías sanitarias para articular el trabajo que realizan de manera complementaria y eficiente. Este modelo está siendo expandido a otras direcciones del MINSA como la Dirección General de Medicamentos, Insumos y Drogas (DIGEMID), el Seguro Integral de Salud (SIS), la Dirección de Intervenciones de Salud Individual y la Dirección General de Estrategias en Salud del MINSA. Este modelo también se ha empezado a aplicar en EsSalud a través del Instituto de Evaluación de Tecnologías en Salud e Investigación (IETSI), con el cual el MINSA coordina la realización de proyectos de colaboración.

En el ámbito internacional, el INS es Centro Colaborador Cochrane y tiene como aliados estratégicos al Instituto de Evaluación Tecnológica en Salud (IETS) de Colombia y el Instituto de Efectividad Clínica y Sanitaria (IECS) de Argentina. Asimismo, participa de la Red de Política Basada en Evidencia (EVIPNet) de la Organización Panamericana de la Salud, la Red Andina de Evaluación de Tecnologías Sanitarias de las Ámericas (REDETSA), la Red Andina de Evaluación de Tecnologías Sanitarias y el Instituto Sudamericano, UNASUR.

De esta manera, el Perú está participando en un proceso mundial y recibiendo el apoyo de organizaciones como EVIP-net en producción de informes de políticas y otros formatos de fácil uso para la síntesis de la investigación y el debate de las opciones políticas, así como actividades de intercambio de información y buenas prácticas entre los países participantes. Un área que aún falta fortalecer en el Perú, es la de procesos de seguimiento y evaluación para documentar el impacto de la política y programas basados en evidencia. La participación en estas redes internacionales va a permitir al Perú fortalecer el desarrollo de mejores políticas y programas en salud.

Contribuciones de autoría: MP, ELG, EG y GH, participaron en la concepción del trabajo. MP y ELG escribieron la primera versión del manuscrito. Todos los autores aportaron a la redacción y revisión crítica del manuscrito y su aprobación final.

Fuente de financiamiento: Instituto Nacional de Salud.

Conflicto de intereses: los investigadores declaran no tener conflictos de interés. 


\section{REFERENCIAS BIBLIOGRÁFICAS}

1. Bosch-Capblanch X, Lavis JN, Lewin S, Atun R, Røttingen JA, 2 Dröschel D, et al. Guidance for Evidence-Informed Policies about Health Systems: Rationale for and Challenges of Guidance Development. PLoS Med. 2012;9(3):e1001185. doi: 10.1371/journal.pmed.1001185.

2. Oxman $A D$, Fretheim $A$, Schünemann HJ, SURE. Improving the use of research evidence in guideline development: introduction. Health Res Policy Syst. 2006;4:12. doi: 10.1186/1478-4505-412

3. Ministerio de Economía y Finanzas del Perú. Programas presupuestales. Diseño, revisión y articulación territorial 2016. Lima: MEF; 2016.

4. Ministerio de Economía y Finanzas del Perú. Directiva para los programas presupuestales en el marco de la programación y formulación del presupuesto del sector público para el año fiscal 2017. Directiva $\mathrm{N}^{\circ} 003-2015$ EF/50.01. Lima: MEF; 2015.

5. Ministerio de Economía y Finanzas del Perú. Evaluación de impacto del Programa Articulado Nutricional sobre la desnutrición crónica de los niños menores de 5 años [Internet]. Lima: MEF; 2014 [citado el 15 de junio de 2016]. Disponible en: https://www. mef.gob.pe/contenidos/presu_publ/ estudios/PAN_final_fusionado.pdf

6. Instituto Nacional de Estadística e Informática. Encuesta Nacional de Hogares sobre Condiciones de Vida y Pobreza 2013 [Internet]. Lima: INEI; 2013 [citado el 15 de junio de 2016]. Disponible en: http://www.ilo.org/ surveydata/index.php/catalog/358

7. Instituto Nacional de Estadística e Informática. Encuesta Demográfica y de Salud Familiar-ENDES [Internet]. Lima: INEI; 2013 [citado el 15 de junio de 2016]. Disponible en: https://www. inei.gob.pe/media/MenuRecursivo/ publicaciones_digitales/Est/Lib1151/ index.html

8. Instituto Nacional de Estadística e Informática. Encuesta Nacional de Programas Estratégicos 2011-2013 [Internet]. Lima: INEI; 2015 [citado el 15 de junio de 2016]. Disponible en: https://www.inei.gob.pe/media/ MenuRecursivo/publicaciones digitales/Est/Lib1190/libro.pdf

9. Instituto Nacional de Estadística e Informática. Encuesta Nacional de Satisfacción de Usuarios en Salud ENSUSALUD 2014. Lima: INEI; 2014.

10. Instituto Nacional de Estadística e Informática. Encuesta a Establecimientos de Salud en la Atención del Control del Crecimiento, Desarrollo y Vacunas en el menor de 1 año 2015. Lima: INEI; 2015.
11. Instituto Nacional de Estadística e Informática. Encuesta para la medición de la línea base de indicadores de prevalencia y riesgo de transmisión de tuberculosis 2011-2012. Lima: INEI, 2012.

12. Perú, Ministerio de Salud. Metodología para la elaboración de guías de práctica clínica. Documento Técnico. Lima: MINSA/DGSP; 2015.

13. Perú, Ministerio de Salud. Norma técnica de salud para la elaboración y uso de guías de práctica clínica. Lima: MINSA/ DGSP; 2015.

14. Perú, Ministerio de Salud. Guía de práctica clínica para el diagnóstico, tratamiento y control de la Diabetes Mellitus Tipo 2 en el primer Nivel de Atención. Lima: MINSA; 2015.

15. Perú, Ministerio de Salud. Directiva Sanitaria para la prevención de anemia mediante la suplementación con micronutrientes y hierro en niñas y niños menores de 36 meses de edad. Resolución Ministerial N ${ }^{\circ}$ 055-2016. Lima: MINSA; 2016.

16. Instituto Nacional de Salud. Evaluación de los efectos adversos de los alimentos genéricamente modificados en la salud humana: revisión de la literatura científica. Nota Técnica 2011-3. Lima: INS/UNAGESP; 2011.

17. Perú, Ministerio del Ambiente. Ley 29811. Ley que establece la moratoria al ingreso y producción de organismos vivos modificados al territorio nacional por un período de 10 años. (9 de diciembre de 2011).

18. Instituto Nacional de Salud. Evaluación económica de las vacunas hepta-, decay trece-valente para la prevención de neumonía asociada al Streptococcus pneumoniae en niños menores de 5 años. Nota Técnica 2011-6. Lima: INS/ UNAGESP; 2011.

19. Mezones-Holguín E, Bolaños-Días R, Fiestas V, Sanabria C, Gutiérrez-Aguado A, Fiestas F, et al. Cost-effectiveness analysis of pneumococcal conjugate vaccines in preventing pneumonia in Peruvian children. J Infect Dev Ctries. 2014;8(12):1552-62. doi: $10.3855 /$ jidc. 5855 .

20. Instituto Nacional de Salud. Estudio de costo efectividad de las vacunas decay trece-valente para la prevención de enfermedad asociada a Streptococcus pneumoniae en niños. Nota Técnica 2012-3. Lima: INS/UNAGESP; 2012.

21. Mezones-Holguin E, Canelo-Aybar C, Clark A, Janusz C, Jauregui B, EscobedoPalza $S$, et al. Cost-effectiveness analysis of 10- and 13- valent pneumococcal conjugate vaccines in Peru. Vaccine. 2015;33 Suppl 1:A154-66. doi: 10.1016/j.vaccine.2014.12.039.

22. Perú, Ministerio de Salud. Resolución Ministerial No 510-2013: norma técnica de salud que establece el Esquema Nacional de Vacunación. Lima: MINSA; 2013.

23. Instituto Nacional de Salud. Eficacia de las vacunas bivalente y cuadrivalente del Virus de Papiloma Humano (VPH) para prevenir lesiones cérvico-uterinas pre-malignas o malignas. Nota Técnica 3. Lima: INS/UNAGESP; 2013.

24. Vargas K, Tejada R, Benites-Zapata V, Mezones-Holguín E, Bolaños-Díaz R, Hernández A. Eficacia de las vacunas contra el virus papiloma humano en la prevención de lesiones oncogénicas diferentes a cáncer de cuello uterino: revisión sistemática. Lima: INS/ UNAGESP; 2014.

25. Tejada R, Vargas K, Benites-Zapata V, Mezones-Holguín E, Bolaños R, Fiestas $\mathrm{F}$, et al. Eficacia de la vacuna contra el virus del papiloma humano en la prevención de lesiones no oncológicas: revisión sistemática y tema-análisis. Lima; INS/ UNAGESP; 2014.

26. Instituto Nacional de Salud. Costo efectividad de la vacuna contra el papiloma virus humano. Nota Técnica 2011-7. Lima: INS; 2011.

27. Solari L, Hijar G, Zavala R, Ureta JM. Revisión sistemática de evaluaciones económicas de fármacos antivirales para el tratamiento de la hepatitis B crónica: una revisión sistemática. Rev Peru Med Exp Salud Pública. 2010;27(1):68-79.

28. Perú, Ministerio de Salud. Plan Nacional Concertado de Salud. Lima: MINSA; 2007.

29. Perú, Ministerio de Salud. Norma técnica de planificación familiar: Norma Técnica 032-MINSA/DGSP-V.01. Lima: MINSA; 2005.

30. Suárez VJ, Zavala R, Ureta JM, Hijar G, Lucero J, Pachas P. Efecto del levonorgestrel como anticonceptivo oral de emergencia en la ovulación, el endometrio y los espermatozoides. Rev Peru Med Exp Salud Pública. 2010;27(2):222-30.

31. Carmona G, Osorio V, Daza L, Portocarrero J. Percepciones sobre la vacunación contra la rabia silvestre en población Awajún de la provincia de Condorcanqui, Amazonas Perú. Rev Saúde Coletiva. 2016;26(1):201-24.

32. Perú, Ministerio de Salud. Oficina General de comunicaciones. Plan de Comunicaciones de Rabia 2013 [Internet]. Lima: MINSA; 2013 [citado el 15 de junio de 2016]. Disponible en: http://www.minsa.gob.pe/portada/ Especiales/2013/rabia/matcom/ plancomunicacional.pdf

\section{Correspondencia: Marina Piazza}

Dirección: Calle Cápac Yupanqui 1400.

Jesús María. Lima 11, Perú

Teléfono: (511) 748-1111 anexo 2113

Correo electrónico: mpiazza@ins.gob.pe 